ISSN 1855-3966 (printed edn.), ISSN 1855-3974 (electronic edn.)

ARS MATHEMATICA CONTEMPORANEA 19 (2020) 189-208

https://doi.org/10.26493/1855-3974.2033.974

(Also available at http://amc-journal.eu)

\title{
Divergence zero quaternionic vector fields and Hamming graphs
}

\author{
Jasna Prezelj * \\ Fakulteta za matematiko in fiziko, Jadranska 19, 1000 Ljubljana, Slovenija, and \\ UP FAMNIT, Glagoljaška 8, 6000 Koper, Slovenija, and \\ IMFM, Jadranska 19, Ljubljana, Slovenija \\ Fabio Vlacci ${ }^{\dagger}$ \\ DiSPeS Università di Trieste Piazzale Europa 1, Trieste, Italy \\ Dedicated to the memory of Marjan Jerman.
}

Received 3 July 2019, accepted 1 July 2020, published online 13 November 2020

\begin{abstract}
We give a possible extension of the definition of quaternionic power series, partial derivatives and vector fields in the case of two (and then several) non commutative (quaternionic) variables. In this setting we also investigate the problem of describing zero functions which are not null functions in the formal sense. A connection between an analytic condition and a graph theoretic property of a subgraph of a Hamming graph is shown, namely the condition that polynomial vector field has formal divergence zero is equivalent to connectedness of subgraphs of Hamming graphs $H(d, 2)$. We prove that monomials in variables $z$ and $w$ are always linearly independent as functions only in bidegrees $(p, 0),(p, 1),(0, q),(1, q)$ and $(2,2)$.
\end{abstract}

Keywords: Quaternionic power series, bidegree full functions, Hamming graph, linearly independent quaternionic monomials.

Math. Subj. Class. (2020): 30G35, 15A03, 05C10

* The first author was partially supported by research program P1-0291 and by research projects J1-7256 and J1-9104 at Slovenian Research Agency. Part of the paper was written when the first author was visiting the DiMaI at University of Florence and she wishes to thank this institution for its hospitality.

$\dagger$ The second author was partially supported by Progetto MIUR di Rilevante Interesse Nazionale PRIN 2010 11 Varietà reali e complesse: geometria, topologia e analisi armonica. The research that led to the present paper was partially supported by a grant of the group GNSAGA of Istituto Nazionale di Alta Matematica "F: Severi".

E-mail addresses: jasna.prezelj@fmf.uni-lj.si (Jasna Prezelj), fvlacci@units.it (Fabio Vlacci)

()( This work is licensed under https://creativecommons.org/licenses/by/4.0/ 


\section{Introduction}

Complex holomorphic vector fields with divergence zero represent an important tool for the description of the groups of volume preserving automorphisms of $\mathbb{C}^{n}$ with $n>1$ (we refer the reader to [1] and [2] for a thorough description of this topic). In this paper we investigate generalizations of complex holomorphic vector fields in the quaternionic setting, and for this purpose we restrict our research to mappings represented by convergent quaternionic power series.

We introduce an alternative definition of partial derivative, namely as a first order approximation (which is not linear) and using this new notion of partial derivatives we define the corresponding divergence in the quaternionic setting. We show that quaternionic vector fields with divergence zero are bidegree full (see Section 2.2 for definition) and that the divergence zero condition on quaternionic vector fields is equivalent to finding connected subgraphs of Hamming graphs. The paper is structured as follows: Section 2 contains the description of our setting with basic definitions and notions, such as partial derivatives and divergence. Moreover, bidegree full functions are introduced together with some basic facts about Hamming graphs. Section 3 is devoted to vector fields and their properties, in particular it contains the main result, Theorem 3.4, on quaternionic vector fields with divergence zero and explains the connection between divergence zero vector fields and Hamming graphs. In Section 4 we prove the theorem on linear independence of monomials.

\section{Preliminaries}

\subsection{Convergent quaternionic power series}

In this section we introduce the basic concepts and notions to deal with generalizations of complex holomorphic power series in the quaternionic setting.

We denote by $\mathbb{H}$ the algebra of quaternions, $\mathbb{H}=\left\{z=x_{0}+x_{1} i+x_{2} j+x_{3} k, x_{0}, \ldots\right.$, $\left.x_{3} \in \mathbb{R}\right\}$, where $i, j, k$ are imaginary units satisfying $i^{2}=j^{2}=k^{2}=-1, i j=k, j k=$ $i, k i=j$. Denote by $\mathbb{S}$ the sphere of imaginary unit quaternions, i.e. the set of quaternions $I$ such that $I^{2}=-1$; notice that for a quaternion $z$ we have $z^{2}=x_{0}^{2}-x_{1}^{2}-x_{2}^{2}-x_{3}^{2}+$ $2 x_{0}\left(x_{1} i+x_{2} j+x_{3} k\right)$, therefore the condition $z^{2}=-1$ implies $z=x_{1} i+x_{2} j+x_{3} k$ and $-x_{1}^{2}-x_{2}^{2}-x_{3}^{2}=-1$. Given any nonreal quaternion $z$, there exist (and are uniquely determined) an imaginary unit $I$, and two real numbers $x, y$ (with $y>0$ ) such that $z=$ $x+I y$. With this notation, the conjugate of $z$ will be $\bar{z}:=x-I y$. Each imaginary unit $I$ generates (as a real algebra) a copy of a complex plane denoted by $\mathbb{C}_{I}$. We call such a complex plane a slice.

A product of nonzero quaternionic coefficients and the variables $z, w$ of degree $d$ is called a generalized quaternionic monomial of degree $d$. Let $\mathbb{H}_{d}[z, w]$ denote the set of all finite sums of generalized quaternionic monomials of degree $d$, which we call generalized quaternionic homogenous polynomials of degree $d$. For example, the generalized quaternionic polynomial $a_{0} z a_{1} w a_{2} w a_{3}+b_{0} z^{2} b_{1} w b_{3}+c_{0} w c_{1} z c_{2} w c_{3}$ belongs to $\mathbb{H}_{3}[z, w]$. Let

$$
\mathbb{H}[z, w]:=\bigoplus_{d \geq 0} \mathbb{H}_{d}[z, w]
$$

be the ring of generalized quaternionic polynomials in the variables $z, w$ over the quaternions. We consider polynomials $P \in \mathbb{H}[z, w]$ as formal (left and right) linear combinations. 
It turns out (see Section 4) that there are several polynomials defining the same polynomial function. We therefore identify a given polynomial function $P$ with the equivalence class $[P]$ of all polynomials defining the same function. The set of all polynomial functions coincides with real polynomials in 8 variables with quaternionic coefficients (see [3]).

We consider the right-submodule $\mathbb{H}_{\text {rhs }}[z, w]$ of $\mathbb{H}[z, w]$ which consists of all generalized quaternionic polynomials whose generalized monomials have coefficients on the right-hand side. To be precise, given the multiindex $\alpha=\left(\alpha_{1}, \ldots, \alpha_{d}\right) \in\{0,1\}^{d}$, called a word on letters 0,1 , we define the length of $\alpha$ to be $|\alpha|:=\sum_{l=1}^{d} \alpha_{i}$. Then we put

$$
(z, w)^{\alpha}:=\left(z^{\alpha_{1}} w^{1-\alpha_{1}}\right) \cdots\left(z^{\alpha_{d}} w^{1-\alpha_{d}}\right) .
$$

For integers $p, q \geq 0, p+q=d$, denote by $\alpha^{p, q}$ a multiindex with $\left|\alpha^{p, q}\right|=p$. There are $\left(\begin{array}{l}d \\ p\end{array}\right)$ such multiindices. We call the pair $(p, q)$ a bidegree. The (pure) monomials of degree $d$ can be written in the form

$$
(z, w)^{\alpha^{p, q}}
$$

and hence define

$$
\begin{aligned}
& \mathbb{H}_{\mathrm{rhs},(p, q)}[z, w]:=\left\{P_{p, q}(z, w)=\sum_{\substack{\alpha^{p, q},\left|\alpha^{p, q}\right|=p}}(z, w)^{\alpha^{p, q}} c_{\alpha^{p, q}} ; c_{\alpha^{p, q}} \in \mathbb{H}\right\} \\
& \mathbb{H}_{\mathrm{rhs}, d}[z, w]:=\left\{P_{p, q}(z, w)=\sum_{\substack{\alpha^{p, q},\left|\alpha^{p, q}\right|=p}}(z, w)^{\alpha^{p, q}} c_{\alpha^{p, q}} ; c_{\alpha^{p, q}} \in \mathbb{H}, p+q=d\right\}
\end{aligned}
$$

so that $\mathbb{H}_{\text {rhs }}[z, w]=\oplus_{d \geq 0} \mathbb{H}_{\text {rhs }, d}[z, w]$.

Our basic assumption on regularity, for the definition of the class of quaternionic series we are interested in, is that any such a series $f$

$$
f(z, w)=\sum_{p, q \geq 0} \sum_{\lambda \in \Lambda_{p, q}} f_{p, q, \lambda}(z, w)
$$

converges absolutely on $\mathbb{H}^{2}$. Notice that absolute convergence implies uniform convergence on compact sets of $\mathbb{H}^{2}$. The notation $f_{p, q, \lambda}(z, w) \in \mathbb{H}_{d}[z, w]$ stands for generalized monomials containing $p$ copies of $z$ and $q$ copies of $w$ with $p+q=d$ and the sets $\Lambda_{p, q}$ are supposed finite. The set of all such series $f$ will be denoted by $\mathcal{H}[z, w]$. Putting

$$
f_{d}(z, w):=\sum_{p=0}^{d} \sum_{\lambda \in \Lambda_{p, d-p}} f_{p, d-p, \lambda}(z, w),
$$

any $f \in \mathcal{H}[z, w]$ also has a homogenous expansion $f(z, w)=\sum_{d \geq 0} f_{d}(z, w)$. Uniform convergence on compact sets of $\mathbb{H}^{2}$ means that given any $\varepsilon>0$ and a compact set $K \subset \mathbb{H}^{2}$, there exists a natural number $d_{\varepsilon, K}$ such that for any generalized polynomial of the form

$$
P(z, w)=\sum_{d=0}^{d_{\varepsilon, K}} f_{d}(z, w)+\sum_{d>d_{\varepsilon, K}} \sum_{p=0}^{d} \sum_{\lambda \in \Lambda_{p, d-p}^{\prime} \subset \Lambda_{p, d-p}} f_{p, d-p, \lambda}(z, w),
$$

the uniform estimate $|f(z, w)-P(z, w)|_{K}<\varepsilon$ holds. Let the norm of the term $f_{p, q, \lambda}$ be $\left|f_{p, q, \lambda}(z, w)\right|=|z|^{p}|w|^{q} c_{p, q, \lambda}$ (with $c_{p, q, \lambda} \geq 0$ ) and define $c_{p, q}:=\sum_{\lambda \in \Lambda_{p, q}} c_{p, q, \lambda}$. The 
absolute convergence at the point $\left(z_{0}, w_{0}\right)$ in the domain of definition of $f$ means that

$$
\sum_{p, q \geq 0} \sum_{\lambda \in \Lambda_{p, q}}\left|f_{p, q, \lambda}\left(z_{0}, w_{0}\right)\right|=\sum_{p, q \geq 0}\left|z_{0}\right|^{p}\left|w_{0}\right|^{q} c_{p, q}<\infty
$$

and implies uniform convergence on compact sets of $B\left(0,\left|z_{0}\right|\right) \times B\left(0,\left|w_{0}\right|\right)$.

Any series $f \in \mathcal{H}[z, w]$ uniquely defines a function of two quaternionic variables, but as in the case of polynomials, there are many series defining the same function. We say that two quaternionic series are equivalent if each of them defines the same quaternionic function. This is an equivalence relation, and so we identify the function $f$ with the corresponding equivalence class $[f]$ of all series in $\mathcal{H}[z, w]$ defining the same function. To avoid too many notations, we will say that a given function belongs to $\mathcal{H}[z, w]$ if it has a series representative in $\mathcal{H}[z, w]$. By abuse of notation, if $f \in \mathcal{H}[z, w]$, we also denote by $[f]$ the set of all series which determine the same function. Since uniqueness of the power series for a function $f$ is not granted (see next paragraphs and (2.2)), the absolute convergence of a chosen power series for a given function $f \in \mathcal{H}[z, w]$ is not a consequence of uniform convergence on compact sets, as in the complex or real case, and has to be additionally required. In the sequel we focus our attention on the right $\mathbb{H}$-module $\mathcal{H}_{\text {rhs }}[z, w]$ in $\mathcal{H}[z, w]$ of (absolutely convergent) power series with coefficients on the right. We extend all the above definitions also to series of three or more variables. Notice that in $\mathbb{H}_{\mathrm{rhs}, d}\left[z_{1}, z_{2}, \ldots, z_{n}\right]$ there are $n^{d}$ different (pure) monomials. If we assume only uniform convergence of series in $\mathcal{H}_{\mathrm{rhs}}\left[z_{1}, z_{2}, \ldots, z_{n}\right]$, given an uniformly convergent series $f\left(z_{1}, z_{2}, \ldots, z_{n}\right)=\sum_{d>0} f_{d}\left(z_{1}, z_{2}, \ldots, z_{n}\right)$, for $R>0, \varepsilon>0$ there is a $d_{0} \in \mathbb{N}$ such that for each $d \geq d_{0}$ and $p=\left(p_{1}, \ldots, p_{n}\right) \in \mathbb{N}_{0}^{d}$ with $|p|=\sum_{1}^{n} p_{1}=d$, where $p$ denotes the multiindex, whose $j$ th element $p_{j}$ is the total degree of $z_{j}$ in the corresponding monomial, the estimate $\left|f_{p, \lambda}\left(z_{1}, z_{2}, \ldots, z_{n}\right)\right|<\varepsilon$ holds on the ball $B^{n}(0, R) \subset \mathbb{H}^{n}$. As a consequence, on the ball $B^{n}(0, R /(n+1))$, we have the estimate $\left|f_{p, \lambda}\left(z_{1}, z_{2}, \ldots, z_{n}\right)\right|<$ $\varepsilon /(n+1)^{|p|}$ (with $|p|=\sum_{1}^{n} p_{1}=d$ ), so that for $\left(z_{1}, z_{2}, \ldots, z_{n}\right) \in B^{n}(0, R /(n+1)$ ) we have

$$
\sum_{|p|=d}\left|f_{p, \lambda}\left(z_{1}, z_{2}, \ldots, z_{n}\right)\right|<\varepsilon\left(\frac{n}{n+1}\right)^{d}
$$

which implies that the series $f\left(z_{1}, z_{2}, \ldots, z_{n}\right)=\sum_{d \geq 0} f_{d}\left(z_{1}, z_{2}, \ldots, z_{n}\right)$ is not just uniformly but also absolutely convergent. Once more, we observe that, in general, in $\mathcal{H}\left[z_{1}, z_{2}, \ldots, z_{n}\right]$ one has to assume absolute convergence for a proper definition of series, since the number of different generalized monomials can grow faster than exponentially; for example, if the polynomial $P$ is as in (2.2), then the sums $\sum_{k=0}^{m} P\left(z, a_{k}\right)$, with $a_{k} \in \mathbb{H}$, are identically 0 for any $m \in \mathbb{N}$ and they contain $6 m$ different generalized monomials. Let us mention another right-submodule of $\mathcal{H}_{\text {rhs }}[z, w]$, namely, the submodule of slice-regular functions in the sense of Ghiloni-Perotti (see [5]), denoted by $\mathcal{H}_{\mathrm{GP}}[z, w]$. It is generated by (pure) monomials of the form $z^{k} w^{l}, k, l \in \mathbb{N}_{0}$, with this precise order, so any element of $\mathcal{H}_{\mathrm{GP}}[z, w]$ has a unique power series expansion and uniform convergence on compact sets in $\mathbb{H}$ implies absolute convergence. Slice-regular functions in the sense of GhiloniPerotti can be also seen as the kernel of a suitable partial differential operator. Notice that $\mathcal{H}_{\mathrm{GP}}[z, w] \subset \mathcal{H}_{\mathrm{rhs}}[z, w] \subset \mathcal{H}[z, w]$.

Unfortunately, also in $\mathcal{H}_{\mathrm{rhs}}[z, w]$ there are several power series which define the same function. In general the monomials of a given bidegree are not (right) linearly independent as functions. As far as we know, very little is known about this question except for linear 
independence of monomials of bidegrees $(p, 1)$ and $(1, q)$ as proved in [6, Proposition 2.4]. In Section 4 we prove that monomials of bidegrees $(p, 0),(p, 1),(1, q),(0, q)$ and $(2,2)$ are linearly independent but monomials of bidegree $(3,2)$ (and all other bidegrees) are not necessarily: since the square of the commutator of $z$ and $w$ is real, i.e. $[z, w]^{2} \in \mathbb{R}$, the polynomial of bidegree $(3,2)$,

$$
P(z, w)=-z^{2} w z w+z^{2} w^{2} z+z w z^{2} w-z w^{2} z^{2}-w z^{2} w z+w z w z^{2}=\left[[z, w]^{2}, z\right]
$$

is identically zero as a function but it is not (formally) equal to the null polynomial. Therefore, even here there is no one-to-one correspondence between power series and functions. However, as we will see, this fact does not affect the generality of the problem we are interested in (see also Remark 3.8 and Example 4.4). We realized that there exists a submodule $\mathcal{H}^{\mathrm{BF}}[z, w]$ in $\mathcal{H}_{\mathrm{rhs}}[z, w]$ which gives rise to vector fields with nice analytic properties, but these vector fields could not in general be detected using just analytic tools, due to the fact that we are not able to describe formal properties of the series defining the zero class $[0]$. Nevertheless, it turns out that these vector fields have representatives in their corresponding classes of power series with specific symmetry properties and for them all the results stated are valid within a given bidegree up to adding a polynomial which defines the identically-zero function. Example 4.4 is a special case where analytic conditions imply the existence of this special type of representatives in the classes of power series and these representatives are unique.

We remark that $\mathcal{H}_{\mathrm{GP}}[z, w]$ contains, as a particular case, the right submodule of sliceregular functions in one variable denoted by $\mathcal{S R}$ as introduced in [4] (see also the monograph [3]): it is the class $\mathcal{H}_{\text {rhs }}[z]:=\mathcal{H}_{\text {rhs }}[z, 1]$. Vaguely speaking it is defined to be the class of functions $f: \mathbb{H} \rightarrow \mathbb{H}$ such that the limit

$$
\lim _{h \rightarrow 0} h^{-1}(f(z+h)-f(z))
$$

exists if $h$ and $z$ belong to the same slice. These functions turn out to be quaternionic analytic and their power expansions are unique.

In general, there is no standard way of introducing a notion of (partial) derivative for quaternionic functions (see for instance [4, 5]). For example, for the slice-regular function $f(z)=z^{2} a$ the limit of the differential quotient

$$
\lim _{h \rightarrow 0} h^{-1}(f(z+h)-f(z))=\lim _{h \rightarrow 0}\left(h^{-1} z h+z+h\right) a
$$

does not exist unless $h$ and $z$ belong to the same slice.

We introduce new differential operators $\widehat{\partial}_{z}, \widehat{\partial}_{w}: \mathcal{H}[z, w] \rightarrow \mathcal{H}[z, w, h]$, which can be interpreted as partial derivatives for a convergent power series as in (2.1) with respect to each of the variables $z, w$ in a given direction $h$.

Definition 2.1. For a function $f \in \mathcal{H}[z, w]$ and $z_{0}, w_{0}, h_{0} \in \mathbb{H}$ we define the quaternion $\widehat{\partial}_{z} f\left(z_{0}, w_{0}\right)\left[h_{0}\right]$ to be the limit

$$
\widehat{\partial}_{z} f\left(z_{0}, w_{0}\right)\left[h_{0}\right]:=\lim _{t \rightarrow 0} \frac{1}{t}\left(f\left(z_{0}+t h_{0}, w_{0}\right)-f\left(z_{0}, w_{0}\right)\right), \quad t \in \mathbb{R},
$$

or equivalently

$$
f\left(z_{0}+t h_{0}, w_{0}\right)-f\left(z_{0}, w_{0}\right)=t \widehat{\partial}_{z} f\left(z_{0}, w_{0}\right)\left[h_{0}\right]+o(|t|) ;
$$


similarly

$$
\widehat{\partial}_{w} f\left(z_{0}, w_{0}\right)\left[h_{0}\right]:=\lim _{t \rightarrow 0} \frac{1}{t}\left(f\left(z_{0}, w_{0}+t h_{0}\right)-f\left(z_{0}, w_{0}\right)\right), \quad t \in \mathbb{R},
$$

defines $\widehat{\partial}_{w} f\left(z_{0}, w_{0}\right)\left[h_{0}\right]$. The function $\widehat{\partial}_{z} f$ in three variables $(z, w, h)$ is then defined to be

$$
\left(\widehat{\partial}_{z} f\right)(z, w, h):=\widehat{\partial}_{z} f(z, w)[h],
$$

and similarly

$$
\left(\widehat{\partial}_{w} f\right)(z, w, h):=\widehat{\partial}_{w} f(z, w)[h] .
$$

We use the notation $\widehat{\partial}_{z} f(z, w)[h], \widehat{\partial}_{w} f(z, w)[h]$ also to denote the resulting functions of three variables in order to emphasize the special role the variable $h$ plays.

Both the operators $\widehat{\partial}_{z}, \widehat{\partial}_{w}$ are additive and right- $\mathbb{H}-$ linear, namely

$$
\begin{aligned}
\widehat{\partial}_{z}(f(z, w) a+g(z, w) b)[h] & =\widehat{\partial}_{z} f(z, w)[h] a+\widehat{\partial}_{z} g(z, w)[h] b, \\
\widehat{\partial}_{w}(f(z, w) a+g(z, w) b)[h] & =\widehat{\partial}_{w} f(z, w)[h] a+\widehat{\partial}_{w} g(z, w)[h] b .
\end{aligned}
$$

The resulting functions are additive and real-homogenous in the variable $h$, but not linear in $h$. Furthermore, the Leibniz rule holds. In the language of analysis on manifolds, for a fixed $h$, the partial derivative $\widehat{\partial}_{z} f(z, w)[h]$ is the Lie derivative of the function $f$ along the constant vector field $X=(h, 0)$ evaluated at $(z, w)$ and $\widehat{\partial}_{w} f(z, w)[h]$ is the Lie derivative of the function $f$ along the constant vector field $X=(0, h)$ evaluated at $(z, w)$. In practice, for polynomial function represented by a polynomial, each of the operators $\widehat{\partial}_{z}, \widehat{\partial}_{w}$ acts by replacing one occurrence of the prescribed variable at a time in each monomial of $f_{d}$ with $h \in \mathbb{H}$ as in the following example

$$
\widehat{\partial}_{z}\left(z w z^{2} w a\right)[h]=\left(h w z^{2} w+z w h z w+z w z h w\right) a .
$$

If $\left|f_{p, q, \lambda}(z, w)\right|=|z|^{p}|w|^{q} c_{p, q, \lambda}$, then we can estimate

$$
\left|\widehat{\partial}_{z} f_{p, q, \lambda}(z, w)[h]\right| \leq p|z|^{p-1}|w|^{q}|h| c_{p, q, \lambda}
$$

and $\left|\widehat{\partial}_{w} f_{p, q, \lambda}(z, w)[h]\right| \leq q|z|^{p}|w|^{q-1}|h| c_{p, q, \lambda}$, which, in view of the assumed absolute convergence of the power series, implies that the power series can be differentiated term by term. Therefore operators $\widehat{\partial}_{z}, \widehat{\partial}_{w}$ are well-defined as mappings from quaternionic analytic functions of two variables to quaternionic analytic functions of three variables. This motivates the following definition of partial derivatives for series:

Definition 2.2. Given a series $f \in \mathcal{H}[z, w]$,

$$
f(z, w)=\sum_{p, q \geq 0} \sum_{\lambda \in \Lambda_{p, q}} f_{p, q, \lambda}(z, w)
$$

the series $\widehat{\partial}_{z} f$ is defined as

$$
\left(\widehat{\partial}_{z} f\right)(z, w, h):=\sum_{p, q \geq 0} \sum_{\lambda \in \Lambda_{p, q}} \widehat{\partial}_{z} f_{p, q, \lambda}(z, w)[h] .
$$

The operator $\widehat{\partial}_{w}$ is defined similarly. Note that the operators $\widehat{\partial}_{z}, \widehat{\partial}_{w}$ map series in $\mathcal{H}[z, w]$ to series in $\mathcal{H}[z, w, h]$. 
We also use the notation $\widehat{\partial}_{z} f(z, w)[h]$ for the series to indicate the special role the variable $h$ plays.

Linearity of the derivation implies that if a function is represented by two different series $f$ and $g$, then also the series $\widehat{\partial}_{z} f(z, w)[h]$ and $\widehat{\partial}_{z} g(z, w)[h]$ represent the same function.

The following result motivates the introduction of the differential operators $\widehat{\partial}_{z}, \widehat{\partial}_{w}$.

Lemma 2.3. Let $f \in \mathcal{H}_{\mathrm{rhs}}[z, w]$ be a series. If $\widehat{\partial}_{z} f(z, w)[h]$ is the null-series, then $f(z, w)$ is (formally) independent of $z$ and so is also the corresponding function. An analogous result holds for $w$.

Proof. It suffices to prove the first assertion for polynomials $P_{(p, q)}$ of bidegree $(p, q)$ for each $(p, q)$. We proceed by induction on $q$. For $q=0$ and $P_{(p, 0)}(z, w)=z^{p} c_{p}$ we have

$$
\widehat{\partial}_{z} P_{(p, 0)}(z, w)[h]=\left(h z^{p-1}+z h z^{p-2}+\cdots+z^{p-1} h\right) c_{p}=0
$$

formally, so $c_{p}=0$. Moreover, by [6, Proposition 2.4] the same holds if $\widehat{\partial}_{z} P_{(p, 0)}(z, w)[h]=$ 0 as a function. If $q>0$ write

$$
P_{(p, q)}(z, w)=z P_{(p-1, q)}(z, w)+w P_{(p, q-1)}(z, w)
$$

and then the formal identity

$\widehat{\partial}_{z} P_{(p, q)}(z, w)[h]=h P_{(p-1, q)}(z, w)+z \widehat{\partial}_{z} P_{(p-1, q)}(z, w)[h]+w \widehat{\partial}_{z} P_{(p, q-1)}(z, w)[h]=0$ implies

$$
P_{(p-1, q)}(z, w)=0, \widehat{\partial}_{z} P_{(p-1, q)}(z, w)[h]=0 \quad \text { and } \quad \widehat{\partial}_{z} P_{(p, q-1)}(z, w)[h]=0
$$

formally. By induction hypothesis, $\widehat{\partial}_{z} P_{(p, q-1)}(z, w)[h]$ being formally 0 implies $P_{(p, q-1)}(z, w)=w^{q-1} c_{q-1}$, so $P_{(p, q)}=w^{q} c_{q-1}$.

Remark 2.4. In analogy to the one variable case one could also define the (differential) operator

$$
\widetilde{\partial}_{z} f(z, w):=\widehat{\partial}_{z} f(z, w)[1] .
$$

In short, the operator $\widetilde{\partial}_{z}$ replaces one occurrence of the variable $z$ at a time with 1 . This operator is a derivation. Using the notation from the above Lemma, the expression $\widetilde{\partial}_{z} P_{(p, q)}(z, w)$ is a polynomial of bidegree $(p-1, q)$ (similarly for $w$ ). Furthermore, this operator coincides with the corresponding (Cullen) derivative, when $f$ is a slice-regular function (see [4]).

However, a result like the one in Lemma 2.3 does not hold when considering $\widetilde{\partial}_{z}$ instead of $\widehat{\partial}_{z}$. Indeed,

$$
\widetilde{\partial}_{z}(z w-w z)=w-w=0
$$

but the neither the series $f(z, w)=z w-w z$ nor the corresponding function do not depend on $w$ only. 


\subsection{Bidegree full series}

For $p, q$ positive integers, consider the series

$$
S_{p, q}(z, w):=\sum_{\substack{\alpha^{p, q}, \alpha^{p, q} \mid=p \\ p+q=d}}(z, w)^{\alpha^{p, q}} .
$$

It is clear that $S_{p, q}(z, w)=S_{q, p}(w, z)$. We also have this important identity

$$
\widehat{\partial}_{z} S_{p+1, q}(z, w)[h]=\widehat{\partial}_{w} S_{p, q+1}(z, w)[h] .
$$

If $z$ and $w$ commute, then $S_{p, q}(z, w)=\left(\begin{array}{c}p+q \\ p\end{array}\right) z^{p} w^{q}$.

Definition 2.5. We define

$$
\mathbb{H}_{d}^{B F}[z, w]:=\left\{\sum_{p+q=d} S_{p, q}(z, w) a_{p, q}, a_{p, q} \in \mathbb{H}\right\}
$$

and

$$
\mathbb{H}^{B F}[z, w]:=\bigoplus_{d \geq 0} \mathbb{H}_{d}^{B F}[z, w] .
$$

We say that $\mathbb{H}^{B F}[z, w]$ is the right module of bidegree full (in short BF) polynomials in the variables $z, w$. The equivalence class of BF polynomials is called a bidegree polynomial function. Similarly, we define the right module of bidegree full series to consist of all converging power series of the form

$$
f(z, w)=\sum_{d=0}^{\infty} f_{d}(z, w)
$$

with $f_{d}(z, w) \in \mathbb{H}_{d}^{B F}[z, w]$ and denote it by $\mathcal{H}^{B F}[z, w]$. The equivalence class of a BF series is called a bidegree full function.

The following result shows that bidegree full polynomials form an interesting class of polynomials.

Lemma 2.6. For any real number $\mu$ and any $d \in \mathbb{N}$, the polynomial

$$
(z-\mu w)^{d}:=\overbrace{(z-\mu w) \cdots(z-\mu w)}^{d \text { times }}
$$

is bidegree full. If

$$
P(z, w)=\sum_{d=0}^{l} \sum_{\substack{p, q \geq 0, p+q=d}} S_{p, q}(z, w) a_{p, q}
$$

is a bidegree full polynomial of degree $d$, then it also has a decomposition

$$
P(z, w)=\sum_{d=0}^{l} \sum_{p+q=d}\left(\sum_{n=0}^{d}(z-\mu w)^{d} r_{p, d}(n)\right) a_{p, q}, \quad \text { with } \quad r_{p, d}(n) \in \mathbb{R} .
$$


Proof. Indeed, from direct calculations, it follows that

$$
(z-\mu w)^{d}=(z-\mu w) \cdots(z-\mu w)=\sum_{\substack{p, q \geq 0, p+q=d}} S_{p, q}(z, w)(-\mu)^{q} .
$$

The second statement follows from the fact (proved in [2] by induction on $d$ with an argument which applies to our setting) that the polynomials $\left\{x^{d},(x-1)^{d}, \ldots,(x-d)^{d}\right\}$ form a basis of real polynomials of order less or equal to $d$ and consequently polynomials $z^{d},(z-w)^{d}, \ldots,(z-d w)^{d}$ form a basis of $\mathbb{H}_{d}^{B F}[z, w]$

The term $(z-\mu w)^{d}$ is well-defined also for $\mu \in \mathbb{H}$. But the equality

$$
\widehat{\partial}_{w}(z-\mu w)^{d}=-\mu \widehat{\partial}_{z}(z-\mu w)^{d}
$$

holds if and only if $\mu \in \mathbb{R}$.

Remark 2.7. As a consequence of Lemma 2.6, from any $F \in \mathcal{S R}$, in the variable $u$

$$
F(u)=\sum_{d \geq 0} u^{d} a_{d}
$$

one gets a bidegree full series by replacing $u$ with $z-\mu w, \mu \in \mathbb{R}$ namely

$$
f(z, w)=\sum_{d \geq 0}(z-\mu w)^{d} a_{d} \in \mathcal{H}^{B F}[z, w] .
$$

\subsection{Basics on Hamming graphs}

Since the monomials we are dealing with are described by words on two letters, the Hamming graphs are natural objects to associate with such monomials.

Definition 2.8. Given $d, q \in \mathbb{N}$, the graph $(V, E)$ is a Hamming graph $H(d, q)$ if the set of vertices $V$ consists of all words of length $d$ on $q$ different letters and there is an edge $e\left(v_{1}, v_{2}\right) \in E$ between two vertices $v_{1}, v_{2}$ if they differ in precisely one letter.

The Hamming graph $H(d, q)$ is, equivalently, the Cartesian product of $d$ complete graphs $K_{q}$. We are interested in Hamming graphs on two letters, 0,1 , i.e. on hypercubes. A layer $L_{p}, 0 \leq p \leq d$, is a set of vertices which contain $p$ copies of 1 . It is easy to see that the following result holds:

Lemma 2.9. Any two subsequent layers of the hypercube form a connected subgraph.

Proof. The case $d=1$ is trivial since it consists of letters 0 and 1 and an edge connecting them. Assume that $d>1$ and take $p \in\{0, \ldots, d-1\}$. Let $L_{p+1}$ and $L_{p}$ be two subsequent layers, and let $\alpha, \tilde{\alpha} \in L_{p+1}$ differ for one transposition of indices 0 and 1 on positions $l, m$. Without loss of generality we assume that $l=1$ and $m=2$. We may also assume that

$$
\alpha=01 \alpha_{1} \text { and } \tilde{\alpha}=10 \alpha_{1} \text {. }
$$

Define

$$
\beta=00 \alpha_{1} .
$$

Since $\alpha$ and $\beta$ differ in precisely one letter, there is an edge between $\alpha$ and $\beta$ and of course also an edge between $\beta$ and $\tilde{\alpha}$, so there exist a path between any two vertices in $L_{p+1}$, since all other multiindices in $L_{p+1}$ are permutations of letters of $\alpha$. By the same reason there exist a path connecting any two vertices in $L_{p}$ which proves the lemma. 


\section{Quaternionic vector fields}

In this section, using the partial derivatives $\widehat{\partial}_{z}, \widehat{\partial}_{w}$, we define an operator divergence for quaternionic vector fields in two variables. We show that there is a large class of vector fields with good properties of analyticity.

Definition 3.1. Given the series $f, g \in \mathcal{H}[z, w]$, then $X=(f, g)$ is called a vector field in $\mathbb{H}^{2}$, in short we write $X \in \mathcal{V H}$. If $f, g \in \mathcal{H}_{\text {rhs }}[z, w]$, then we write $X \in \mathcal{V H}_{\text {rhs }}$. In particular, we say that a vector field $X=(f, g)$ is bidegree full (in short BF) if $f, g$ are bidegree full and we use the notation $X \in \mathcal{V} \mathcal{H}^{B F}$. A vector field $X=(f, g)$ defines a vector mapping $[X]:=([f],[g]): \mathbb{H}^{2} \rightarrow \mathbb{H}^{2}$.

We assume from now on that the vector fields under consideration belong to $\mathcal{V} \mathcal{H}_{\text {rhs }}$. Next we introduce the following

Definition 3.2. Given the vector field $X=(f, g) \in \mathcal{V} \mathcal{H}_{\text {rhs }}$, we define the operator Div by

$$
\operatorname{Div} X(z, w)[h]:=\widehat{\partial}_{z} f(z, w)[h]+\widehat{\partial}_{w} g(z, w)[h],
$$

where the partial differential operators are used in the sense of (2.3). A vector field $X(z, w)$ has divergence zero if $\operatorname{Div} X(z, w)[h]$ is the null series.

Clearly for a vector field, divergence zero implies divergence zero as a function.

Example 3.3. The vector field $X(z, w)=\left(z w+w z,-w^{2}\right)$ has divergence zero, since

$$
\operatorname{Div}\left(z w+w z,-w^{2}\right)[h]=h w+w h-(h w+w h)=0
$$

and the divergence of the vector field $Y(z, w)=X(z, w)+\left(0,\left[[z, w]^{2}, z\right]\right)=(z w+$ $\left.w z,-w^{2}+\left[[z, w]^{2}, z\right]\right)$ is

$$
\begin{aligned}
\operatorname{Div}\left(z w+w z,-w^{2}+\left[[z, w]^{2}, z\right]\right)[h]=h w & +w h-(h w+w h) \\
& +[[z, h][z, w], z]+[[z, w][z, h], z] .
\end{aligned}
$$

This shows that $\operatorname{Div} Y$ is not a null-series, but $\operatorname{Div} Y$, considered as a vector mapping, vanishes identically.

The vector field $\left(z^{2} w,-z w^{2}\right)$ does not have divergence zero:

$$
\operatorname{Div}\left(z^{2} w,-z w^{2}\right)[h]=(h z+z h) w-z(h w+w h)=h z w-z w h \neq 0
$$

and also $[h z w-z w h] \neq 0$. By identity (2.5) any vector field $(z-\mu w)^{d}(\mu, 1), \mu \in \mathbb{R}$ has divergence zero. Such vector fields are called shear vector fields and they generate a 1-parameter family of automorphisms of $\mathbb{H}^{2}$, namely

$$
\Phi_{t}(z, w)=(z, w)+t(z-\mu w)^{d}(\mu, 1) a, \quad a \in \mathbb{H}, t \in \mathbb{R}
$$

called shears. In the complex analytic case by a famous result due to Andersen (see [1]) every volume preserving automorphism of $\mathbb{C}^{2}$ (these are holomorphic automorphisms $f: \mathbb{C}^{2} \rightarrow \mathbb{C}^{2}$ with determinant $\left.\operatorname{det} J f(z, w)=1\right)$ is approximable by a finite composition of shears. In search for analogous results in the quaternionic setting, it is then necessary to 
prove that any polynomial divergence zero vector field is generated by a shear vector field. Because of identity (2.4), any vector field

$$
X_{p, q}(z, w)=\left(S_{p+1, q}(z, w),-S_{p, q+1}(z, w)\right)
$$

has divergence zero. It can be shown using Lemma 2.6 that every vector field $X_{p, q}$ is a sum of shear vector fields. The interested reader can find the details in [6]. The next theorem shows that any divergence zero vector field is generated by such vector fields $X_{p, q}$.

Theorem 3.4. Let $X=(f, g)$ be a vector field with divergence zero, then $f$ and $g$ are bidegree full.

Remark 3.5. Example 4.4 shows that for any vector field $X$ with components of bidegrees $(3,2)$ and $(2,3)$, the condition $\operatorname{Div} X(z, w)[h]=0$ as a function of three variables implies that the mapping representing the vector field $X$ has a bidegree full representative.

Corollary 3.6. If $X$ is a vector field with divergence zero, then $X$ is of the form $X=$ $\sum X_{p, q} a_{p, q}, a_{p, q} \in \mathbb{H}$ with $X_{p, q}$ as in (3.1).

Before proceeding to the proof, let us show an example with vector fields of the form $X(z, w)=(f(z, w), g(z, w))=\left(z^{2} w a_{1}+z w z a_{2}+w z^{2} a_{3},-w^{2} z b_{1}-w z w b_{2}-z w^{2} b_{3}\right)$. We first calculate the partial derivatives separately.

$$
\begin{aligned}
& \widehat{\partial}_{z} f(z, w)[h]=(z h w+h z w) a_{1}+(h w z+z w h) a_{2}+(w h z+w z h) a_{3}, \\
& \widehat{\partial}_{w} g(z, w)[h]=-(w h z+h w z) b_{1}-(h z w+w z h) b_{2}-(z w h+z h w) b_{3} .
\end{aligned}
$$

The sum of the partial derivatives is zero if and only if monomials of the same type cancel out, for example we have conditions $z h w\left(a_{1}-b_{3}\right)=0$ and $h z w\left(a_{1}-b_{2}\right)=0$ which imply $a_{1}=b_{3}$ and $a_{1}=b_{2}$ and similarly for other terms. We represent these equalities by means of a bipartite graph on $\left\{a_{1}, a_{2}, a_{3}\right\} \cup\left\{b_{1}, b_{2}, b_{3}\right\}$ in which there is an edge between $a_{i}$ and $b_{j}$ if and only if they are equal. The graph is given in Figure 1.

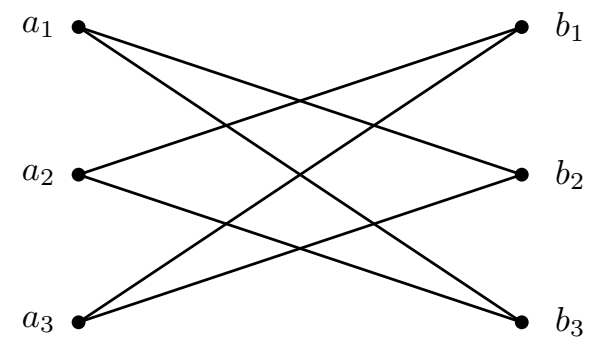

Figure 1: Bipartite graph.

Proof of Theorem 3.4. Let $f(z, w)=\sum f_{p, q}(z, w), g(z, w)=\sum g_{p, q}(z, w)$ be the decompositions of series $f$ and $g$ with respect to the bidegrees. Then $X=(f, g)$ has divergence zero if and only if

$$
\widehat{\partial}_{z} f_{p+1, q}(z, w)[h]+\widehat{\partial}_{w} g_{p, q+1}(z, w)[h]=0 .
$$


Let $\mathcal{A}=\left\{\alpha \in\{0,1\}^{d+1},|\alpha|=p+1\right\}$ and $\mathcal{B}=\left\{\beta \in\{0,1\}^{d+1},|\beta|=p\right\}$. Write

$$
\begin{aligned}
& f_{p+1, q}(z, w)=\sum_{\alpha \in \mathcal{A}}(z, w)^{\alpha} A_{\alpha} \\
& g_{p, q+1}(z, w)=-\sum_{\beta \in \mathcal{B}}(z, w)^{\beta} B_{\beta} .
\end{aligned}
$$

The monomials in the sum $\widehat{\partial}_{z} f_{p+1, q}(z, w)[h]$ (and similarly $\left.\widehat{\partial}_{z} g_{p, q+1}(z, w)[h]\right)$ are of the following form:

$$
(z, w)^{\alpha_{1}} h(z, w)^{\alpha_{2}} A_{\alpha}
$$

where $\alpha=\alpha_{1} 1 \alpha_{2}$. For any such $\alpha$ there is exactly one $\beta$, namely

$$
\beta=\alpha_{1} 0 \alpha_{2}
$$

such that in the sum $\widehat{\partial}_{w}(z, w)^{\beta} B_{\beta}(h)$ there is the monomial of the same type (but multiplied by a different constant)

$$
-(z, w)^{\alpha_{1}} h(z, w)^{\alpha_{2}} B_{\beta} .
$$

Zero divergence implies that $A_{\alpha}=B_{\beta}$ for any such pair $\alpha, \beta$.

Define a bipartite graph on the vertices $V=\mathcal{A} \cup \mathcal{B}$. There is an edge between a word $\alpha \in A$ and a word $\beta \in B$ iff the word $\beta$ is obtained from the word $\alpha$ by replacing one of the letters 1 by 0 . So by definition, in this particular case, we are considering a subgraph of the Hamming graph $H(d+1,2)$, spanned on edges from the set $\mathcal{A} \cup \mathcal{B}$ which represent two subsequent layers in the corresponding hypercube, $\mathcal{A}=L_{p+1}$ and $\mathcal{B}=L_{p}$. By Lemma 2.9 this subgraph is connected. This implies that all $A_{\alpha}=A$ for some constant $A$ and hence the same holds for all $B_{\beta}$ so all the coefficients are the same and this means that $f_{p+1, q}, g_{p, q+1}$ are bidegree full.

Remark 3.7. We should point out that the analytic condition on a vector field of two quaternionic variables having divergence zero is equivalent to connectedness of subgraphs of a Hamming graph. We could proceed analogously in higher dimensions. In three variables we would consider $H(d, 3)$, graphs on three letters, where $d$ is the degree, but in this case the divergence zero condition translates into looking for cycles of order 3 of a particular form. Its analysis turns out to be more complicated than the two-dimensional case and is not related to connectedness of subgraphs of Hamming graphs. For example, the divergence zero condition for the vector field $X(z, w, u)=(f(z, w, u), g(z, w, u), h(z, w, u))$ in the case

$$
\begin{aligned}
& f(z, w, u)=z w u a_{1}+w z u a_{2}+w u z a_{3}+z u w a_{4}+u z w a_{5}+u w z a_{6}, \\
& g(z, w, u)=w^{2} u b_{1}+w u w b_{2}+u w^{2} b_{3} \\
& h(z, w, u)=w u^{2} c_{1}+u w u c_{2}+u^{2} w c_{3}
\end{aligned}
$$

gives equations $a_{1}+b_{1}+c_{2}=0, a_{2}+b_{1}+c_{1}=0, \ldots$ and they can be represented as a 2 -simplicial complex with 2 -cells being triangles with vertices $\left(a_{1}, b_{2}, c_{2}\right),\left(a_{2}, b_{1}, c_{1}\right)$ and so forth (see Figure 2). 


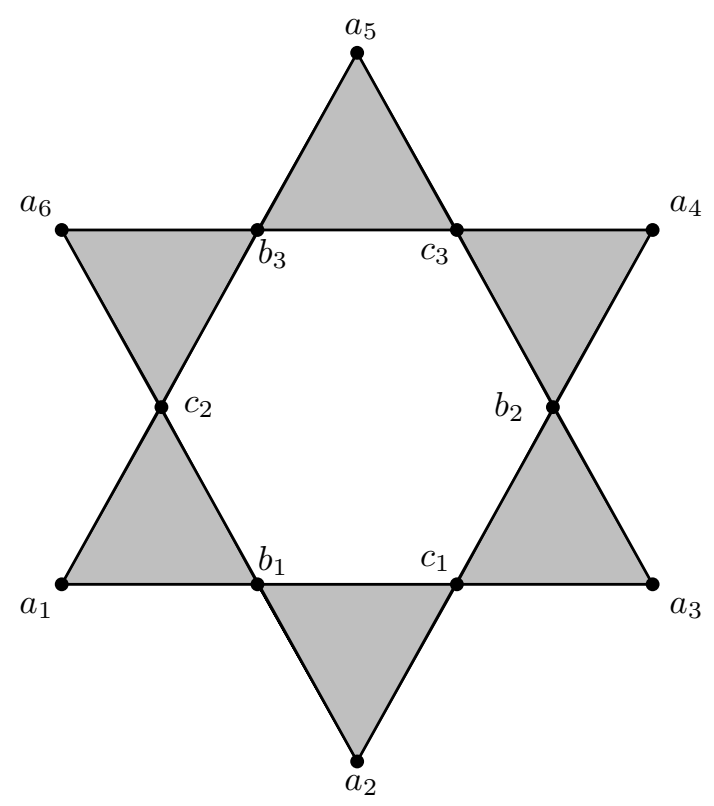

Figure 2: 2-simplicial complex describing the divergence 0 condition for 3 variables.

Remark 3.8. Notice that if we can write a vector mapping as a vector field $X=\left(f_{1}, g_{1}\right)+$ $\left(f_{2}, g_{2}\right)$ such that $\left(f_{1}, g_{1}\right)$ has divergence zero and such that each of $f_{2}$ and $g_{2}$ are not formally 0 but identically equal to 0 as functions, then the flow of $[X]$ coincides with the flow of $\left[\left(f_{1}, g_{1}\right)\right]$. Furthermore, the flow of $\left[\left(f_{2}, g_{2}\right)\right]$ exists and is the identity mapping, so it does not affect the problem of approximating a flow by shears.

\section{Linear independence of monomials}

In this section we consider the problem of linear independence of monomials in $\mathbb{H}_{\mathrm{rhs}}(z, w)$; in particular we exhibit an algorithm for determining linear independence of monomials in $\mathbb{H}_{\mathrm{rhs},(p, q)}[z, w]$. We point out that this approach does not involve the computation of independent monomials in 8 real variables of degree $p$ in the first 4 variables and of degree $q$ in the last 4 variables.

We prove the following result.

Theorem 4.1. Given a bidegree $(p, q)$, the set of all distinct monomials in $\mathbb{H}_{\mathrm{rhs},(p, q)}[z, w]$ is linearly independent if and only if $(p, q)$ equals $(p, 0),(0, q),(p, 1),(1, q)$ or $(2,2)$.

The proof below gives an explicit algorithm for calculating the basis of the kernel of the linear mapping

$$
\mathcal{A}_{p, 2}: \mathbb{H}^{n_{p+2,2}} \rightarrow \mathcal{H}_{\mathrm{rhs}}[z, w], \quad \mathcal{A}_{p, 2}\left(c_{1}, \ldots, c_{n_{p+2,2}}\right)=\sum_{1}^{n_{p+2,2}}(z, w)^{\alpha_{k}} c_{k}
$$

where $\alpha_{k} \in\{0,1\}^{d}$ are all distinct multiindices of length $p,\left|\alpha_{k}\right|=p$, with $k=1, \ldots$, $n_{p+2,2}=\left(\begin{array}{c}p+2 \\ 2\end{array}\right)$. 
Proof. The cases in bidegrees $(p, 0),(0, q)$ are trivial and the cases in bidegrees $(p, 1),(1, q)$ were proved in [6, Proposition 2.4]. Assume that $z \in \mathbb{C}_{I} \backslash \mathbb{R}$ and choose any imaginary unit $J$ orthogonal to $I$. Then we can write $w \in \mathbb{H}$ in the form $z_{0}+z_{1} J$, where $z_{0}, z_{1} \in \mathbb{C}_{I}$ are uniquely determined. This choice of coordinates provides us with a frame which determines the identification $\mathbb{H}=\mathbb{C}_{I} \times \mathbb{C}_{I}$. In other words, if $w=z_{0}+z_{1} J \simeq\left(z_{0}, z_{1}\right) \in$ $\mathbb{C}_{I} \times \mathbb{C}_{I}$, then, since $z J=J \bar{z}$, we have

$$
w^{2}=z_{0}^{2}-\left|z_{1}\right|^{2}+\left(\overline{z_{0}} z_{1}+z_{0} z_{1}\right) J
$$

similarly

$$
[z, w]=z_{1}(z-\bar{z}) J \quad \text { and } \quad[z, w]^{2}=-\left|(z-\bar{z}) z_{1}\right|^{2} .
$$

We recall that the polynomial introduced in (2.2) is precisely $P(z, w)=\left[[z, w]^{2}, z\right]$.

We begin with polynomial $w^{2} \in \mathbb{H}_{\mathrm{rhs},(0,2)}[z, w]$ and develop an algorithm for producing monomials in $\mathbb{H}_{\text {rhs,(1,2) }}[z, w]$ which we describe with respect to the above identification and then proceed inductively.

First of all, without loss of generality, we (may and will) assume that $z$ is unitary, so $z^{-1}=\bar{z}$. Let $A_{0}=B_{0}=C_{0}=\left\{w^{2}\right\}$. Define the sets $A_{1}=\left\{z w^{2}\right\}, B_{1}=\{w z w\}, C_{1}=$ $\left\{w^{2} z\right\}$. The monomial in $A_{1}$ was obtained by adding $z$ to the monomial $w^{2}$ on the left hand side, the one in $B_{1}$ by adding one $z$ after the first $w$ of the monomial $w^{2}$ and the monomial in $C_{1} z$ was obtained by adding a $z$ on the right hand side of the monomial $w^{2}$.

If $w=z_{0}+z_{1} J$ (and then $w^{2}=z_{0}^{2}-\left|z_{1}\right|^{2}+\left(\overline{z_{0}} z_{1}+z_{0} z_{1}\right) J$ ) we have

$$
\begin{aligned}
A_{1} \ni z w^{2} & =z\left(z_{0}^{2}-\left|z_{1}\right|^{2}+\left(\overline{z_{0}} z_{1}, z_{0} z_{1}\right) J\right) \bar{z} z \\
& =\left(z_{0}^{2}-\left|z_{1}\right|^{2}+\left(z^{2} \overline{z_{0}} z_{1}+z^{2} z_{0} z_{1}\right) J\right) z=f_{1}\left(z, z_{0}, z_{1}\right) z, \\
B_{1} \ni w z w & =\left(z_{0}+z_{1} J\right) z\left(z_{0}+z_{1} J\right) \bar{z} z \\
& =\left(\left(z_{0}^{2}-\bar{z}^{2}\left|z_{1}\right|^{2}+\left(\overline{z_{0}} z_{1}+z^{2} z_{0} z_{1}\right) J\right) z=f_{2}\left(z, z_{0}, z_{1}\right) z,\right. \\
C_{1} \ni w^{2} z & =\left(z_{0}^{2}-\left|z_{1}\right|^{2}+\left(\overline{z_{0}} z_{1}, z_{0} z_{1}\right) J\right) z=f_{3}\left(z, z_{0}, z_{1}\right) z .
\end{aligned}
$$

We identify $w^{2}$ with the vector $\left(z_{0}^{2},-\left|z_{1}\right|^{2}, \overline{z_{0}} z_{1}, z_{0} z_{1}\right) \in \mathbb{C}_{I}^{4}$ and identify the function $f_{1}$ with the vector $u_{1}=\left(z_{0}^{2},-\left|z_{1}\right|^{2}, z^{2} \overline{z_{0}} z_{1}, z^{2} z_{0} z_{1}\right) \in \mathbb{C}_{I}^{4}, f_{2}$ with the vector $u_{2}=$ $\left(z_{0}^{2},-\bar{z}^{2}\left|z_{1}\right|^{2}, \overline{z_{0}} z_{1}, z^{2} z_{0} z_{1}\right) \in \mathbb{C}_{I}^{4}$ and the function $f_{3}$ with the vector $u_{3}=\left(z_{0}^{2},-\left|z_{1}\right|^{2}\right.$, $\left.\overline{z_{0}} z_{1}, z_{0} z_{1}\right) \in \mathbb{C}_{I}^{4}$. We notice that $u_{1}$ is obtained from $u_{3}$ by multiplying the first two components by 1 and the last two by $z^{2}$, i.e. $u_{1}=u_{3} *\left(1,1, z^{2}, z^{2}\right)$, where $*$ denotes the componentwise multiplication in $\mathbb{C}_{I}^{4}$ defined as follows: if $(a, b, c, d) \in \mathbb{C}_{I}^{4}$ and $(x, y, u, v) \in \mathbb{C}_{I}^{4}$ then

$$
(a, b, c, d) *(x, y, u, v):=(a x, b y, c u, d v) .
$$

Notice that the componentwise multiplication $*$ in $\mathbb{C}_{I}^{4}$ is commutative, i.e.

$$
(x, y, u, v) *(a, b, c, d)=(a, b, c, d) *(x, y, u, v)
$$

for any $(a, b, c, d) \in \mathbb{C}_{I}^{4}$ and $(x, y, u, v) \in \mathbb{C}_{I}^{4}$ and has no zero divisors.

Similarly $u_{2}=u_{3} *\left(1, \bar{z}^{2}, 1, z^{2}\right)$ and $u_{3}=u_{3} *(1,1,1,1)$. Consider a quaternionic (right-hand side) null linear combination of the monomials which generates $\mathbb{H}_{\text {rhs, (1,2) }}[z, w]$, namely $z w^{2} a+w z w b+w^{2} z c=0$ with $a, b, c \in \mathbb{H}$.

In terms of the vectors $u_{1}, u_{2}, u_{3}$, we can write the same null linear combination as

$$
\begin{aligned}
& u_{3} *\left(1,1, z^{2}, z^{2}\right) z a+u_{3} *\left(1, \bar{z}^{2}, 1, z^{2}\right) z b+u_{3} *(1,1,1,1) z c= \\
& \quad=u_{3} *\left[\left(1,1, z^{2}, z^{2}\right) z a+\left(1, \bar{z}^{2}, 1, z^{2}\right) z b+(1,1,1,1) z c\right]=0 .
\end{aligned}
$$


If we write $a=a_{0}+a_{1} J, b=b_{0}+b_{1} J c=c_{0}+c_{1} J$ (according to the adopted frame) and look at the first component in (4.1), since $u_{3} \neq 0$, we get the equation

$$
\begin{aligned}
z\left(a_{0}+a_{1} J\right)+z\left(b_{0}+b_{0} J\right)+z\left(c_{0}+c_{1} J\right) & =z\left(\left(a_{0}+a_{1} J+b_{0}+b_{0} J+c_{0}+c_{1} J\right)\right. \\
& =z\left[\left(a_{0}+b_{0}+c_{0}\right)+\left(a_{1}+b_{1}+c_{1}\right) J\right]=0
\end{aligned}
$$

which implies $a_{0}+b_{0}+c_{0}=0$ and $a_{1}+b_{1}+c_{1}=0$. From the vanishing of the second component in (4.1) we get the equation

$$
\begin{array}{rl}
1 \cdot z\left(a_{0}+a_{1} J\right)+\bar{z}^{2} & z\left(b_{0}+b_{0} J\right)+z\left(c_{0}+c_{1} J\right) \\
& =z\left(a_{0}+a_{1} J\right)+z \bar{z}^{2}\left(b_{0}+b_{1} J\right)+z\left(c_{0}+c_{1} J\right) \\
& =z\left[\left(a_{0}+a_{1} J\right)+\bar{z}^{2}\left(b_{0}+b_{1} J\right)+\left(c_{0}+c_{1} J\right)\right] \\
& =z\left[\left(a_{0}+\bar{z}^{2} b_{0}+c_{0}\right)+\left(a_{1}+b_{1} \bar{z}^{2}+c_{1}\right) J\right]=0
\end{array}
$$

which implies $a_{0}+\bar{z}^{2} b_{0}+c_{0}=0$ and $\left(a_{1}+\bar{z}^{2} b_{1}+c_{1}\right) J=0$. From the vanishing of the third component in (4.1) we get the equation

$$
\begin{aligned}
z^{2} J z\left(a_{0}+a_{1} J\right)+ & J z\left(b_{0}+b_{0} J\right)+J z\left(c_{0}+c_{1} J\right) \\
& =J z\left(\bar{z}^{2}\left(a_{0}+a_{1} J\right)+\left(b_{0}+b_{1} J\right)+\left(c_{0}+c_{1} J\right)\right. \\
& =J z\left[\left(\bar{z}^{2} a_{0}+b_{0}+c_{0}\right)+\left(\bar{z}^{2} a_{1}+b_{1}+c_{1}\right) J\right)=0
\end{aligned}
$$

which implies $\bar{z}^{2} a_{0}+b_{0}+c_{0}=0$ and $\bar{z}^{2} a_{1}+b_{1}+c_{1}=0$. Here we can replace $\bar{z}^{2}$ with $z^{2}$ since we are allowed to plug in any $z \in \mathbb{C}_{I}$, in particular we can plug in $\bar{z}$ and use the fact that $\overline{\bar{z}}=z$. From the vanishing of the last component in (4.1) we get the equation

$$
\begin{aligned}
z^{2} J z\left(a_{0}+a_{1} J\right)+ & z^{2} J z\left(b_{0}+b_{1} J\right)+J z\left(c_{0}+c_{1} J\right) \\
& =J z\left(\bar{z}^{2}\left(a_{0}+a_{1} J\right)+\bar{z}^{2}\left(b_{0}+b_{1} J\right)+\left(c_{0}+c_{1} J\right)\right. \\
& =J z\left[\left(\bar{z}^{2} a_{0}+\bar{z}^{2} b_{0}+c_{0}\right)+\left(\bar{z}^{2} a_{1}+\bar{z}^{2} b_{1}+c_{1}\right) J\right]=0
\end{aligned}
$$

which implies $\bar{z}^{2} a_{0}+\bar{z}^{2} b_{0}+c_{0}=0$ and $\bar{z}^{2} a_{1}+\bar{z}^{2} b_{1}+c_{1}=0$. Also in these equations, one can substitute $\bar{z}$ with $z .^{1}$ In the vectorial version, we can write the above-given equations as

$$
\begin{aligned}
& \left(1,1, z^{2}, z^{2}\right) a_{0}+\left(1, \bar{z}^{2}, 1, z^{2}\right) b_{0}+(1,1,1,1) c_{0}=0 \\
& \left(1,1, z^{2}, z^{2}\right) a_{1}+\left(1, \bar{z}^{2}, 1, z^{2}\right) b_{1}+(1,1,1,1) c_{1}=0 .
\end{aligned}
$$

Therefore, the linear dependence of the monomials $z w^{2}, w z w$ and $w^{2} z$ (generators of $\left.\mathbb{H}_{\mathrm{rhs},(1,2)}[z, w]\right)$ is equivalent to the linear dependence of the vector functions $X_{1}(z)=$ $\left(1,1, z^{2}, z^{2}\right), X_{2}(z)=\left(1, \bar{z}^{2}, 1, z^{2}\right)$ and $X_{3}(z)=(1,1,1,1)$. In this case, the vector functions $X_{1}(z), X_{2}(z)$ and $X_{3}(z)$ are evidently linearly independent and so are the monomials $z w^{2}, w z w$ and $w^{2} z$.

To generalize the formalization of the above ideas we introduce the operator $\operatorname{ad}_{z}$ and adopt the identification $w^{2} \simeq\left(z_{0}^{2},-\left|z_{1}\right|^{2}, \overline{z_{0}} z_{1}, z_{0} z_{1}\right) \in \mathbb{C}_{I}^{4}$. For $q \in \mathbb{H} \backslash\{0\},|q|=1$, let

\footnotetext{
${ }^{1}$ In all equations we can plug in the variable $\bar{z}$ instead of $z$ and we can consider the conjugated equation. Then the linear independence in question is equivalent to (one of) the above equations with real coefficients.
} 
$\operatorname{ad}_{q}(w):=q w \bar{q}$. This transformation represents a rotation in $\mathbb{H}$ which keeps fixed the slice which contains $q$. Notice that if $z \in \mathbb{C}_{I} \backslash \mathbb{R}$ and $w=z_{0}+z_{1} J$ (with $z_{0}, z_{1} \in \mathbb{C}_{I}$ ), then

$$
\operatorname{ad}_{z}(w)=z_{0}+z_{1} z^{2} J
$$

and

$$
z w^{2}=\left(\operatorname{ad}_{z}(w)\right)^{2} z=\operatorname{ad}_{z}\left(w^{2}\right) z, \quad \operatorname{ad}_{z}\left(\operatorname{ad}_{z}(w)\right)=\operatorname{ad}_{z^{2}}(w)
$$

Then

$$
\begin{aligned}
& \operatorname{ad}_{z}\left(w^{2}\right)=z w^{2} \bar{z} \simeq\left(z_{0}^{2},-\left|z_{1}\right|^{2}, z^{2} \overline{z_{0}} z_{1}, z^{2} z_{0} z_{1}\right) \\
& =\left(z_{0}^{2},-\left|z_{1}\right|^{2}, \overline{z_{0}} z_{1}, z_{0} z_{1}\right) *\left(1,1, z^{2}, z^{2}\right), \\
& w \operatorname{ad}_{z}(w)=w z w \bar{z} \simeq\left(z_{0}^{2},-\bar{z}^{2}\left|z_{1}\right|^{2}, \overline{z_{0}} z_{1}, z^{2} z_{0} z_{1}\right) \\
& =\left(z_{0}^{2},-\left|z_{1}\right|^{2}, \overline{z_{0}} z_{1}, z_{0} z_{1}\right) *\left(1, \bar{z}^{2}, 1, z^{2}\right), \\
& w^{2} \operatorname{ad}_{z}(1)=w^{2} \simeq\left(z_{0}^{2},-\left|z_{1}\right|^{2}, \overline{z_{0}} z_{1}, z_{0} z_{1}\right) \\
& =\left(z_{0}^{2},-\left|z_{1}\right|^{2}, \overline{z_{0}} z_{1}, z_{0} z_{1}\right) *(1,1,1,1) .
\end{aligned}
$$

Define the functions $\varphi_{z}, \psi_{z}, \mathrm{id}_{z}: \mathbb{C}_{I}^{4} \rightarrow \mathbb{C}_{I}^{4}$ by

$$
\begin{aligned}
\varphi_{z}[(a, b, c, d)] & =(a, b, c, d) * X_{1}(z), \quad \psi_{z}[(a, b, c, d)]=(a, b, c, d) * X_{2}(z) \\
\operatorname{id}_{z}[(a, b, c, d)] & =(a, b, c, d) * X_{3}(z) .
\end{aligned}
$$

Since $\varphi_{z}, \psi_{z}$ and $\mathrm{id}_{z}$ are linear in $\mathbb{C}_{I}^{4}$ and can be represented by diagonal matrices, we identify the maps with the diagonals of the corresponding matrices: $\varphi_{z} \cong\left(1,1, z^{2}, z^{2}\right)=$ $X_{1}(z), \psi_{z} \cong\left(1, \bar{z}^{2}, 1, z^{2}\right)=X_{2}(z)$, and $\mathrm{id}_{z} \cong(1,1,1,1)=X_{3}(z)$. In this sense the functions $\varphi_{z}, \psi_{z}$ and $\mathrm{id}_{z}$ are linearly independent since $X_{1}(z), X_{2}(z)$ and $X_{3}(z)$ are linearly independent as vectors.

Now one can write the monomial $z w^{2} \in A_{1}$ as $\varphi_{z}[(1,1,1,1)]=: \mathbf{v}_{1}(z)$, the monomial $w z w \in B_{1}$ as $\psi_{z}[(1,1,1,1)]=: \mathbf{v}_{2}(z)$ and the monomial $w^{2} z \in C_{1}$ as $\operatorname{id}_{z}[(1,1,1,1)]=$ : $\mathbf{v}_{3}(z)$. In the sequel the functions like $\mathbf{v}_{1}, \mathbf{v}_{2}, \mathbf{v}_{3}$ will be called vector functions. With this identification we have $A_{1}=\varphi_{z}\left(A_{0}\right), B_{1}=\psi_{z}\left(B_{0}\right)$ and $C_{1}=\operatorname{id}_{z}\left(C_{0}\right)$. Notice that one can also write $A_{0}=A_{0} \cup B_{0} \cup C_{0}$ and $B_{0}=B_{0} \cup C_{0}$.

We proceed by inductive construction and define

$$
A_{p}=\varphi_{z}\left(A_{p-1} \cup B_{p-1} \cup C_{p-1}\right), B_{p}=\psi_{z}\left(B_{p-1} \cup C_{p-1}\right) \quad \text { and } \quad C_{p}=\operatorname{id}_{z}\left(C_{p-1}\right) \text {. }
$$

The set $A_{p}$ contains all monomials, obtained by adding a $z$ on the left hand side to all bidegree $(p-1,2)$ monomials, the set $B_{p}$ is obtained by adding a $z$ after the first $w$ of the monomials in $B_{p-1}$ and in $C_{p-1}$ and the set $C_{p}$ is obtained by adding a $z$ on the right hand side to the monomials in $C_{p-1}$.

Let us describe the sets $A_{p}, B_{p}, C_{p}$ together with the corresponding vector functions and compute the kernels of $\mathcal{A}_{p, 2}$ for $p=2,3$. Notice that with the adopted identifications, it turns out that $C_{p}=\left\{w^{2} z^{p}\right\}$ for any $p \geq 0$ and this implies that the vector function associated with the unique monomial in $C_{p}$ is the same, namely $(1,1,1,1)$ for any $p \geq 0$.

Here we list the sets $A_{p} B_{p}$ and $C_{p}$ (together with the description of monomials as 
vector functions) for $p=2$ :

$$
\begin{aligned}
& \text { vector function } \\
& A_{2}=\left\{\begin{array}{ll}
\mathbf{v}_{1}(z)=\left(1,1, z^{4}, z^{4}\right) & \simeq z^{2} w^{2} \\
\mathbf{v}_{2}(z)=\left(1, \bar{z}^{2}, z^{2}, z^{4}\right) & \simeq z w z w \\
\mathbf{v}_{3}(z)=\left(1,1, z^{2}, z^{2}\right) & \simeq z w^{2} z
\end{array}\right\} \\
& B_{2}=\left\{\begin{array}{ll}
\mathbf{v}_{4}(z)=\left(1, \bar{z}^{4}, 1, z^{4}\right) & \simeq w z^{2} w \\
\mathbf{v}_{5}(z)=\left(1, \bar{z}^{2}, 1, z^{2}\right) & \simeq w z w z
\end{array}\right\} \\
& C_{2}=\left\{\mathbf{v}_{6}(z)=(1,1,1,1) \quad \simeq w^{2} z^{2}\right\} .
\end{aligned}
$$

Notice that each of the components of the vector functions $\mathbf{v}_{k}(z)$ is generated by $\left\{1, \bar{z}^{2}, \bar{z}^{4}\right.$, $\left.z^{2}, z^{4}\right\}$. We look for the functional kernel of the linear mapping $\mathcal{A}_{2,2}\left(c_{1}, \ldots, c_{6}\right)=$ $\sum_{k=1}^{6} \mathbf{v}_{k}(z) c_{k}$ where the vector functions $\mathbf{v}_{k}(z)$ are listed above; in other words we are imposing conditions on $c_{k}$ 's to have $\sum_{k=1}^{6} \mathbf{v}_{k}(z) c_{k} \equiv 0$ as a function of $z$. From the vanishing of the first component we only get one equation, from the vanishing of the second, third and fourth components the (linear) equations are obtained by imposing the vanishing of coefficients in the basis $\left\{1, \bar{z}^{2}, \bar{z}^{4} z^{2}, z^{4}\right\}$. In this way we obtain a homogeneous linear system whose corresponding matrix is

$$
M_{2}=\left(\begin{array}{llllll}
1 & 1 & 1 & 1 & 1 & 1 \\
1 & 0 & 1 & 0 & 0 & 1 \\
0 & 1 & 0 & 0 & 1 & 0 \\
0 & 0 & 0 & 1 & 0 & 0 \\
0 & 0 & 0 & 1 & 1 & 1 \\
0 & 1 & 1 & 0 & 0 & 0 \\
1 & 0 & 0 & 0 & 0 & 0 \\
0 & 0 & 0 & 0 & 0 & 1 \\
0 & 0 & 1 & 0 & 1 & 0 \\
1 & 1 & 0 & 1 & 0 & 0
\end{array}\right) .
$$

The matrix $M_{2}$ has trivial kernel and this proves the linear independence of sets of distinct monomials in $\mathbb{H}_{\mathrm{rhs},(2,2)}[z, w]$. For $p=3$, using the same approach, we get

$$
\begin{aligned}
& \text { vector function monomial }
\end{aligned}
$$

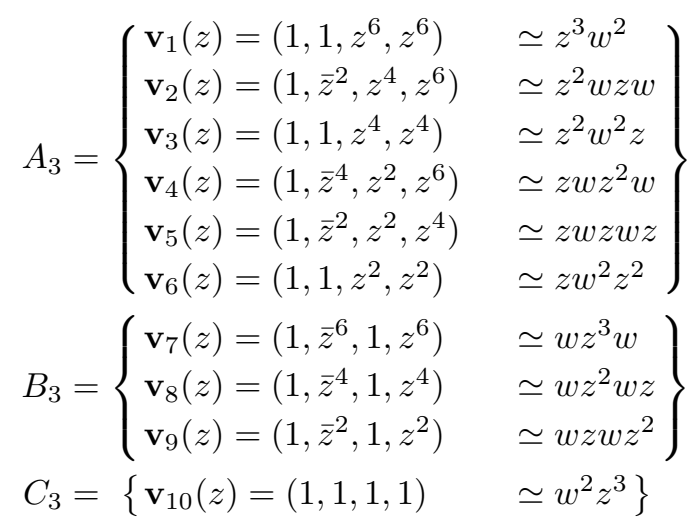


Notice that each of the sets $A_{3}, B_{3}, C_{3}$ contains only linearly independent monomials. We look for the functional kernel of the linear mapping $\mathcal{A}_{3,2}\left(c_{1}, \ldots, c_{10}\right)=\sum_{k=1}^{10} \mathbf{v}_{k}(z) c_{k}$ where the vector functions $\mathbf{v}_{k}(z)$ are listed above; in other words we are imposing conditions on $c_{k}$ 's to have $\sum_{k=1}^{10} \mathbf{v}_{k}(z) c_{k} \equiv 0$ as a function of $z$. We list the equations in the same order as in the previous case. The homogeneous linear system in this case has as corresponding matrix

$$
M_{3}=\left(\begin{array}{llllllllll}
1 & 1 & 1 & 1 & 1 & 1 & 1 & 1 & 1 & 1 \\
1 & 0 & 1 & 0 & 0 & 1 & 0 & 0 & 0 & 1 \\
0 & 1 & 0 & 0 & 1 & 0 & 0 & 0 & 1 & 0 \\
0 & 0 & 0 & 1 & 0 & 0 & 0 & 1 & 0 & 0 \\
0 & 0 & 0 & 0 & 0 & 0 & 1 & 0 & 0 & 0 \\
0 & 0 & 0 & 0 & 0 & 0 & 1 & 1 & 1 & 1 \\
0 & 0 & 0 & 1 & 1 & 1 & 0 & 0 & 0 & 0 \\
0 & 1 & 1 & 0 & 0 & 0 & 0 & 0 & 0 & 0 \\
1 & 0 & 0 & 0 & 0 & 0 & 0 & 0 & 0 & 0 \\
0 & 0 & 0 & 0 & 0 & 0 & 0 & 0 & 0 & 1 \\
0 & 0 & 0 & 0 & 0 & 1 & 0 & 0 & 1 & 0 \\
0 & 0 & 1 & 0 & 1 & 0 & 0 & 1 & 0 & 0 \\
1 & 1 & 0 & 1 & 0 & 0 & 1 & 0 & 0 & 0
\end{array}\right)
$$

whose kernel is spanned by the vector $(0,-1,1,1,0,-1,0,-1,1,0)$. The generator of the kernel represents precisely the polynomial $P(z, w)=\left[[z, w]^{2}, z\right]$ in (2.2).

In the same way one can verify that the kernel of the mapping $\mathcal{A}_{4,2}$ in $\mathbb{H}_{\mathrm{rhs},(4,2)}[z, w]$ is three-dimensional with generators $P_{1}(z, w)=z P(z, w)$ and $P_{2}(z, w)=P(z, w) z$, obtained from the polynomial $P$, and the polynomial

$$
Q(z, w)=[[z, w] z[z, w], z] .
$$

The latter is a zero function since $[z, w] z[z, w]=-\left|(z-\bar{z}) z_{1}\right|^{2} \bar{z}$. The polynomial $Q$ is formally linearly independent from the other two generators since it contains the term $w z^{3} w z$, which does not appear in $P_{1}$ or $P_{2}$. Then the kernel of the mapping $\mathcal{A}_{5,2}$ in $\mathbb{H}_{\mathrm{rhs},(5,2)}[z, w]$ is six-dimensional, generated by $z^{2} P(z, w), z P(z, w) z, P(z, w) z^{2}, z Q(z, w), Q(z, w) z$ and $\left[\left[z^{2}, w\right], z\right]$. By a similar argument as in bidegree $(4,2)$, the first five polynomials are formally linearly independent and the last one contains the term $w z^{4} w z$, which does not appear in the first five polynomials.

In fact it is easy to see that in general the first component of the vector functions in $A_{p}, B_{p}$ and $C_{p}$ is always 1 , whereas in the second component terms containing $1, \bar{z}^{2}, \ldots$, $\bar{z}^{2(p)}$ will appear; similarly, in the third and the fourth component only terms containing $1, z^{2}, \ldots, z^{2 p}$ will show up.

Let us count the number of equations obtained by imposing the vanishing of coefficients of $\mathcal{A}_{p, 2}$. There is only one equation coming from the first component (which is redundant) and the last three components give $\left(2^{2}-1\right)(p+1)$ equations, whereas we have $\left(\begin{array}{c}p+2 \\ 2\end{array}\right)=$ $(p+2)(p+1) / 2$ formally different monomials, so we see that the dimension of the kernel grows quadratically in the bidegree $(p, 2)$. If $p=2$ we have 6 monomials and $9+1$ equations and if $p=3$ there are 10 monomials and $12+1$ equations. If $p=5$ we have for the first time that the number of equations (which is 19) is smaller than the number of monomials (which is 21). 
By a similar procedure one would expect $\left(2^{3}-1\right)(p+1)$ equations for $\left(\begin{array}{c}p+3 \\ 3\end{array}\right)$ monomials in the submodule $\mathbb{H}_{\text {rhs, }(p, 3)}[z, w]$ and so forth, but it turns out the for $q=3$ there are 7 linearly independent monomials of degree 3 in $z_{0}, z_{1}, \bar{z}, \overline{z_{1}}$ in the expression of $w^{3}$, with the first component giving a redundant equation as before, therefore we get less equations.

The same procedure applied to $\mathbb{H}_{\mathrm{rhs},(p, 1)}[z, w]$ is equivalent to looking only at the sets $A_{p}$ and $C_{p}$ and their union, since $B_{p}$ is empty. Moreover in $\mathbb{H}_{\mathrm{rhs},(p, 1)}[z, w]$ the generating monomials have as corresponding vector functions $\left(1,1, z^{2 k}, z^{2 k}\right), k=0, \ldots, p$ and they are obviously linearly independent. This is an alternative proof of Proposition 2.4 in [6].

It is clear that if a set of distinct monomials $\left\{m_{\lambda}(z, w)\right\}_{\lambda \in \Lambda}$ is not linearly independent in the submodule $\mathbb{H}_{\mathrm{rhs},(p, q)}[z, w]$, so the set $\left\{z^{n} m_{\lambda}(z, w)\right\}_{\lambda \in \Lambda}$ is not linearly independent in $\mathbb{H}_{\mathrm{rhs},(p+n, q)}[z, w]$ for each $n \in \mathbb{N}$ and because of symmetry the set $\left\{m_{\lambda}(w, z)\right\}_{\lambda \in \Lambda}$ is not linearly independent in $\mathbb{H}_{\mathrm{rhs},(q, p)}[z, w]$. Putting this together, we see that a subset of all distinct monomials in $\mathbb{H}_{\mathrm{rhs},(3+n, 2+m)}[z, w], m, n \in \mathbb{N}_{0}$ and in $\mathbb{H}_{\mathrm{rhs},(2+n, 3+m)}[z, w]$, $m, n \in \mathbb{N}_{0}$ is not linearly independent.

Since $P(z, w)=\left[[z, w]^{2}, z\right]=0$ as a function and also the polynomial $Q$ of bidegree $(4,2), Q(z, w)=[[z, w] z[z, w], z]$ is identically 0 as a function (as explained in the last section, Equation (4.2)), we conjecture that all zero polynomial functions not formally 0 are obtained from polynomials $P$ and $Q$ after multiplying them by other polynomials and inserting variables $z^{k}$ or $w^{l}$.

Remark 4.2. The described procedure can be interpreted as a complex Fourier series analysis with respect to the complex variables $z, z_{0}$ and $z_{1}$. We could have assumed that all the three variables $z, z_{0}$ and $z_{1}$ are unitary complex numbers, since the modulus is not relevant. In the expansion we considered, there are only 4 generators of the basis of the Fourier series in variables $z_{0}$ and $z_{1}$ and this is reflected in the vector functions having 4 components. With respect to the variable $z$, the number of the basic vector functions in question is $2 p+1$ if bidegree is $(p, 2)$.

Remark 4.3. After applying the partial derivative operator $\widehat{\partial}_{z}$ to the generators of the kernel of $\mathcal{A}_{p, q}$ in $S_{p, q}(z, w)$, one obtains polynomials in tridegree $(p-1, q, 1)$ with respect to variables $z, w, h$, e.g. polynomials with $p-1$ copies $z, q$ copies of $w$ and one $h$. Analogous statement holds for $\widehat{\partial}_{w}$.

Example 4.4. Consider a vector field $X=(f(z, w), g(z, w))$, where $f$ has bidegree $(3,2)$ and $g$ has bidegree $(2,3)$, and let the vector field $Y$ be defined by $Y(z, w)=$ $X(z, w)+(P(z, w) a, \tilde{P}(z, w) b)$, (with $a, b, \in \mathbb{H})$, where $P$ is the bidegree $(3,2)$ polynomial defined in (2.2) and the polynomial $\tilde{P}(z, w)=P(w, z)$ is then a bidegree $(2,3)$ polynomial. Obviously we have $\operatorname{Div} X(z, w)[h]=\operatorname{Div} Y(z, w)[h]$ as a function since $P$ and $\tilde{P}$ are identically 0 as functions. Within this bidegree, the equivalence relation $X \sim Y$ if $[X-Y]=[0]$ means $X-Y=(P a, \tilde{P} b)$ for some choice of $a, b \in \mathbb{H}$. After a careful study of linear independence of monomials in tridegree $(2,2,1)$, i.e. monomials with two copies of $z$-s, two copies of $w$-s and one copy of $h$-which, it should be mentioned, boils down to determining the kernel of a $80 \times 30$ linear system !!! - it turns out that in this particular case, $\operatorname{Div} X(z, w)[h]=0$ as a function if and only if $X=X_{2,2}+(P a, \tilde{P} b)$, which means that the vector mapping has divergence 0 as a function if and only if it has a bidegree full representative in the sense of the above equivalence relation. Examples of bidegree full polynomial vector fields are given in (3.1). 


\section{ORCID iDs}

Jasna Prezelj (DD https://orcid.org/0000-0002-0384-6736

Fabio Vlacci (D) https://orcid.org/0000-0002-6248-8633

\section{References}

[1] E. Andersén, Volume-preserving automorphisms of $\mathbf{C}^{n}$, Complex Variables Theory Appl. 14 (1990), 223-235, doi:10.1080/17476939008814422.

[2] E. Andersén and L. Lempert, On the group of holomorphic automorphisms of $\mathbf{C}^{n}$, Invent. Math. 110 (1992), 371-388, doi:10.1007/bf01231337.

[3] G. Gentili, C. Stoppato and D. C. Struppa, Regular Functions of a Quaternionic Variable, Springer Monographs in Mathematics, Springer, Heidelberg, 2013, doi:10.1007/ 978-3-642-33871-7.

[4] G. Gentili and D. C. Struppa, A new theory of regular functions of a quaternionic variable, $A d v$. Math. 216 (2007), 279-301, doi:10.1016/j.aim.2007.05.010.

[5] R. Ghiloni and A. Perotti, Slice regular functions of several Clifford variables, AIP Conf. Proc. 1493 (2012), 734-738, doi:10.1063/1.4765569.

[6] J. Prezelj and F. Vlacci, On a class of automorphisms in $\mathbb{H}^{2}$ which resemble the property of preserving volume, Math. Nach., in press, arXiv:1810.11412 [math.CO]. 